\title{
Simultaneous Multislice for Accelerating Diffusion MRI in Clinical Neuroradiology Protocols
}

\author{
(D) M.J. Hoch, (D) M. Bruno, (DD. Pacione, (D) Y.W. Lui, (D)E. Fieremans, and (D)T.M. Shepherd
} o- $=$

\begin{abstract}
BACKGROUND AND PURPOSE: Diffusion MR imaging sequences essential for clinical neuroradiology imaging protocols may be accelerated with simultaneous multislice acquisitions. We tested whether simultaneous multislice-accelerated diffusion data were clinically equivalent to standard acquisitions.
\end{abstract}

\begin{abstract}
MATERIALS AND METHODS: In this retrospective study, clinical diffusion sequences obtained before and after implementation of 2-slice simultaneous multislice acceleration and an altered diffusion gradient sampling scheme using the same 3T MRI scanner and 20-channel coil ( $n=25$ per group) were independently and blindly evaluated by 2 neuroradiologists for perceived quality, artifacts, and overall diagnostic utility. Diffusion tractography was performed in 13 patients both with and without 2-slice simultaneous multislice acceleration ( $b=0,1000,2000 \mathrm{~s} / \mathrm{mm}^{2} ; 60$ directions). The corticospinal tract and arcuate fasciculus ipsilateral to the lesion were generated using the same ROIs and then blindly assessed by a neurosurgeon for anatomic fidelity, perceived quality, and impact on surgical management. Tract volumes were compared for spatial overlap.
\end{abstract}

RESULTS: Two-slice simultaneous multislice diffusion reduced acquisition times from 141 to 45 seconds for routine diffusion and from 7.5 to 5.9 minutes for diffusion tractography using 3T MR imaging. The simultaneous multislice-accelerated diffusion sequence was rated equivalent for diagnostic utility despite reductions to perceived image quality. Simultaneous multislice-accelerated diffusion tractography was rated clinically equivalent. Dice similarity coefficients between routine and simultaneous multislice-generated corticospinal tract and arcuate fasciculus tract volumes were 0.78 (SD, 0.03 ) and 0.71 (SD, 0.05), respectively.

CONCLUSIONS: Two-slice simultaneous multislice diffusion appeared clinically equivalent for standard acquisitions and diffusion tractography. Simultaneous multislice makes it feasible to acquire higher angular and $q$-space-resolution diffusion acquisitions required for translating advanced diffusion models into clinical practice.

ABBREVIATIONS: $\mathrm{AF}=$ arcuate fasciculus; $C S T=$ corticospinal tract; $\mathrm{DSC}=$ Dice similarity coefficient; FA $=$ fractional anisotropy; GRAPPA $=$ generalized autocalibrating partially parallel acquisition; SMS = simultaneous multislice

he diffusion-weighted sequence is essential for clinical protocols in head MR imaging and detecting acute ischemic

Received February 3, 2021; accepted after revision March 4.

From the Department of Radiology (M.J.H.), University of Pennsylvania, Philadelphia, Pennsylvania; Departments of Radiology (M.B., Y.W.L., E.F., T.M.S.) and Neurosurgery (D.P.), New York University Langone School of Medicine, New York, New York; and Center for Advanced Imaging Innovation and Research (E.F.), New York, New York.

Timothy Shepherd received research support from the National Institute of Aging (National Institutes of Health 1K23 AG048622-01). This research was supported, in part, by the Center for Advanced Imaging Innovation and Research, a National Institutes of Health-National Institute of Biomedical Imaging and Bioengineering Biomedical technology resource center (grant P41EB017183).

Please address correspondence to Timothy Shepherd, MD/PhD, Department of Radiology, Room 230D, 660 First Ave, New York, NY 10016; e-mail: timothy.shepherd@nyulangone.org; @tim0shepherd; @RVUhound; @elsfi

- Indicates open access to non-subscribers at www.ajnr.org

Indicates article with online supplemental data.

http://dx.doi.org/10.3174/ajnr.A7140 stroke. ${ }^{1}$ Nervous tissue diffusion properties can be informative for other pathologies, including prognosis in lymphoma, ${ }^{2}$ detection of skull lesions, ${ }^{3}$ or early diagnosis of Creutzfeldt-Jakob disease. ${ }^{4}$ Diffusion tractography has improved our understanding of white matter anatomy, like the arcuate fasciculus (AF), the key pathway for language. ${ }^{5}$ Diffusion tractography improves neurosurgical planning and reduces perioperative morbidity for patients with brain tumors ${ }^{6-8}$ but requires high-angular-resolution acquisitions that prolong scan time. During the past 15 years, more advanced diffusion models that account for non-Gaussian water diffusion have further improved our understanding of white matter structure ${ }^{9}$ but require both high-angular-resolution $^{10,11}$ and high $q$-space-resolution ${ }^{12}$ acquisition strategies; these protocols necessitate relatively long scan times that are much harder for patients to tolerate and thus have not been widely implemented clinically. 
Simultaneous multislice (SMS) is a recent innovation that allows concurrently acquiring multiple image slices during each acquisition, ${ }^{13}$ thereby enabling reduced TR and total scan time. Parallel imaging-based SMS uses controlled aliasing with blipped zdirection gradients ${ }^{14,15}$ and then reconstructs images individually using a section generalized autocalibrating partially parallel acquisition (GRAPPA) reconstruction ${ }^{16}$ with little penalty to the image SNR. This technique has become popular in the radiology research community because it supports ever more complex diffusion MR imaging acquisitions for advanced nervous tissue models and representations. ${ }^{17-19}$ Researchers, however, seldom rely on visual interpretation of SMS-diffusion trace images, nor do they depend on the fidelity of diffusion tractography for high-risk clinical decision-making in individual patients. The goal of this study was to evaluate SMS acceleration for clinical neuroradiology diffusion and tractography applications.

\section{MATERIALS AND METHODS}

Initially, volunteers gave consent and were scanned under a prospective, technical development institutional review board (New York University) approval using outpatient 3T MR imaging (Magnetom Skyra; Siemens) with a 20-channel head coil to compare multiple versions of 2-slice SMS-accelerated diffusion sequences to determine an acceptable sequence for common neuroradiology protocols that require trace-weighted and ADC maps, and for a diffusion tractography protocol for patients requiring presurgical planning. The 2-slice SMS-accelerated sequence common to most protocols was then implemented on multiple $1.5 \mathrm{~T}$ and 3T clinical scanners. Diffusion data for tractography still were obtained with both the original and SMS-accelerated sequences until comparison and approval also could be obtained by the referring neurosurgery service. Data analyzed for this report were collected and analyzed retrospectively from the time period around SMS implementation after institutional review board approval of a Health Insurance Portability and Accountability Act-compliant research study.

\section{Clinical Neuroradiology Head Protocols}

Diffusion trace and ADC images from 25 consecutive patients imaged with neuroradiology head protocols using the hospital 3T MR imaging scanner (Magnetom Skyra) with a 20-channel coil were collected from the first day of the month before and after implementation of the 2-slice accelerated SMS diffusion sequence. Consecutive cases were included regardless of reported diagnoses and included inpatients or patients in the emergency department with infarcts, hemorrhage, or brain tumors. The only exclusion criterion was severe motion degradation, but no studies were excluded. There were 2 key changes to the sequence (Online Supplemental Data): First, we used 2-slice SMS acceleration to reduce the TR from 8200 to $3400 \mathrm{~ms}$. Second, the diffusion-sampling scheme was changed by the following: 1) eliminating $b=500 \mathrm{~s} / \mathrm{mm}^{2}$ sampling, 2) increasing gradient directions from 3 to 6 at $b=1000 \mathrm{~s} / \mathrm{mm}^{2}$, and 3 ) reducing averages from 2 to 1 per direction. If we counted $b=0 \mathrm{~s} / \mathrm{mm}^{2}$ images (2 averages), the total diffusion-weighted images were reduced from 14 to 8 (43\% reduction in diffusion volumes acquired). Note that 2-slice SMS TR reductions will always be greater than the resulting total scan time reductions because "dummy" scans are required to reach a steady-state before image acquisition occurs. Overall, these 2 changes reduced scan time from 141 to 45 seconds.

Blinded to the acquisition sequence and alternating side per subject, the senior author used ROIs to extract ADC for the frontal lobe white matter (minor forceps) and frontal horn lateral ventricle CSF (free of the choroid plexus), and to calculate relative SNR for the minor forceps and cerebellar white matter relative to a noise ROI in the same trace-weighted image using previously published methods. ${ }^{20}$ Next, 2 board-certified neuroradiologists blinded to the acquisition sequence independently rated 3 features of the diffusion-weighted trace and ADC images: artifacts (motion, fat suppression, skull base susceptibility, geometric distortion), image quality (resolution, edge sharpness, noise, gray-white junction), and overall diagnostic utility (ability to interpret). Each rating used the same ordinal scale, where 1 was poor, needs recall; 2 was suboptimal, but interpretable; 3 was average, lower range of normal quality; 4 was good, higher range of normal quality; and 5 was excellent. Sample size per group was determined a priori specifically to detect a 0.5 change in the ordinal rating scale in which the predicted mean $=4.0$ (SD, 0.5), $\alpha=.05$, and $\beta \sim 0.95$. ROI and ordinal data were compared by unpaired 2-tailed $t$ tests and MannWhitney-Wilcoxon 2-tailed tests, respectively. Confidence intervals and Cohen $\kappa$ coefficients for interrater reliability were calculated.

\section{Diffusion Tractography Protocol}

Thirteen consecutive brain-mapping cases obtained for neurosurgical planning had both conventional and SMS acquisitions for DTI; this group included 9 glial tumors, 2 seizure foci, 1 cavernous malformation, and 1 epidermoid cyst (mean age, 44 [SD, 36] years, 5 woman. No potential subjects were excluded. Distance documented in the original radiology report between the lesion margin and the visualized corticospinal tract (CST) and/or AF in the ipsilateral hemisphere was $0 \mathrm{~cm}(n=4),<1 \mathrm{~cm}(n=5),<2.5 \mathrm{~cm}$ $(n=3)$, or $\sim 4 \mathrm{~cm}(n=1)$. In this latter patient, tractography was requested because the CST was in closer proximity to the planned surgical corridor than the lesion. One patient had a repeat diffusion scan with SMS acceleration 30 days later for a second procedure.

The shared sequence parameters included $54 \times 3 \mathrm{~mm}$ thick slices with $2.8-\mathrm{mm}$ in-plane resolution $(200-\mathrm{cm}$ FOV, $88 \times 88 \mathrm{ma}-$ trix) with identical diffusion parameters ( 1 average, $b$-values $=0$, $1000,2000 \mathrm{~s} / \mathrm{mm}^{2}, 30$ directions per $b$-value, bipolar gradients). Higher $b$-value data are part of our institutional protocol but may not be considered necessary at other institutions. TR and TE were 7100/95 and 4700/95 ms for standard and 2-slice SMS acquisitions, respectively. Shorter TEs (with resultant shorter TRs and overall scan time) are possible with monopolar diffusion gradients but may be more affected by eddy currents. Volumetric T1, diffusion, and SMS diffusion acquisitions were coregistered in third-party brain mapping software (Brainlab). Two ROIs per tract were drawn on the direction-encoded color fractional anisotropy (FA) maps derived from the standard diffusion acquisitions to generate CST and AF tracts ipsilateral to the lesion ${ }^{21}$ (Online Supplemental Data).

Diffusion data with and without SMS were used separately to generate the tracts while keeping the ROIs constant, thus eliminating manual drawing biases. The minimum tract lengths were 80 and $50 \mathrm{~mm}$ for CST and AF, respectively. The lower limit of FA was 0.15 and 0.12 for CST and AF, respectively. Tractography 
Table 1: Quantitative and qualitative comparison of diffusion trace and ADC parameter maps for clinical patients in the emergency department (single single 3T MRI scanner) ${ }^{\mathrm{a}}$

\begin{tabular}{|c|c|c|c|c|}
\hline Data & Standard $(n=25)$ & SMS $(n=25)$ & 95\% CI for Difference & $P$ Value \\
\hline Age (yr) & $61.5[\mathrm{SD}, 19.5]$ & $63.2[S D, 15.4]$ & NA & $.734^{\mathrm{b}}$ \\
\hline Woman (No.) (\%) & $52 \%(13 / 25)$ & $56 \%(14 / 25)$ & NA & NA \\
\hline ADC, minor forceps $\left(\times 10-3 \mathrm{~mm}^{2} / \mathrm{s}\right)$ & 0.833 [SD, 0.077] & $0.816[S D, 0.073]$ & -0.025 to +0.059 & $.427^{\mathrm{b}}$ \\
\hline $\mathrm{ADC}, \mathrm{CSF}\left(\times 10-^{3} \mathrm{~mm}^{2} / \mathrm{s}\right)$ & $3.032[\mathrm{SD}, 0.099]$ & $3.015[\mathrm{SD}, 0.080]$ & -0.033 to +0.067 & $.508^{\mathrm{b}}$ \\
\hline SNR, minor forceps & $21.4[S D, 14.6]$ & $22.5[S D, 9.6]$ & -7.9 to +5.7 & $.754^{\mathrm{b}}$ \\
\hline SNR, cerebellum & $32.6[\mathrm{SD}, 10.9]$ & $27.3[\mathrm{SD}, 14.8]$ & -1.9 to +12.5 & $.156^{\mathrm{b}}$ \\
\hline Artifacts $^{c}$ & $3.5[\mathrm{SD}, 0.5]$ & $3.7[\mathrm{SD}, 0.5]$ & -0.5 to +0.1 & $.070^{\mathrm{d}}$ \\
\hline Image quality ${ }^{c}$ & $4.0[\mathrm{SD}, 0.7]$ & $3.3[\mathrm{SD}, 0.5]$ & -0.4 to +1.0 & $.001^{\mathrm{d}}$ \\
\hline Diagnostic utility ${ }^{c}$ & $3.8[\mathrm{SD}, 0.4]$ & $3.7[\mathrm{SD}, 0.5]$ & -0.2 to +0.4 & $.187^{\mathrm{d}}$ \\
\hline
\end{tabular}

Note:-NA indicates not applicable

${ }^{a}$ Data are mean (SD), (No.) (\%), or $95 \% \mathrm{Cl}$.

bunpaired, 2-tailed $t$ test.

${ }^{c}$ Average of independent, blinded assessment using an ordinal scale $1-5$ by 2 board-certified neuroradiologists (no adjudication performed).

${ }^{\mathrm{d}}$ Mann-Whitney-Wilcoxon 2-tailed test.

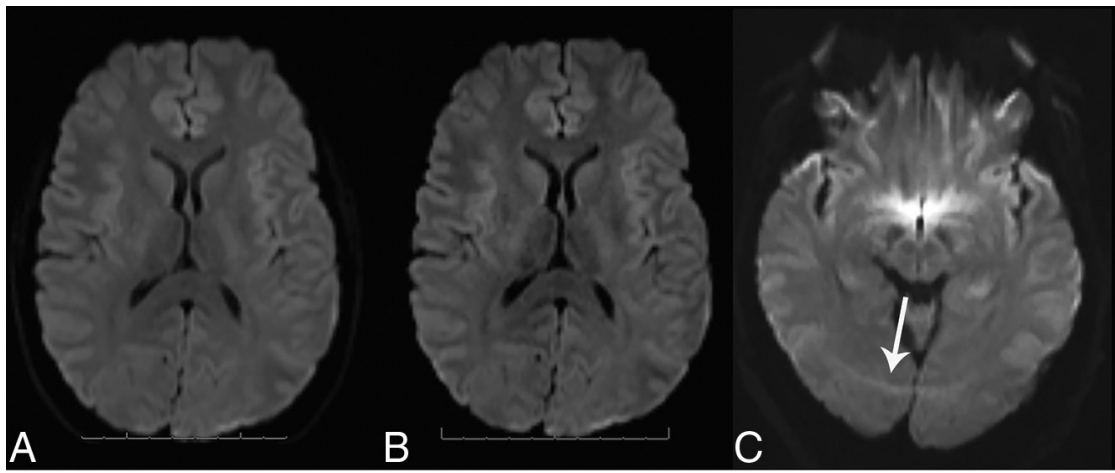

FIG 1. Axial diffusion images from standard $(A)$ and SMS diffusion acquisitions $(B)$ in a clinical patient with headaches. There is no appreciable difference in diagnostic utility, even with a reduction in scan time using SMS. An SMS arc-like scalp artifact (C, arrow) is seen in another clinical patient due to poor fat saturation. With SMS, unsaturated fat signal associated with echo-planar acquisitions can alias into all simultaneously acquired slices.

seed density and turning angle limits have fixed values in the clinical software used. While normally part of our clinical practice, here potential spurious tracts were not edited, nor were FA thresholds adjusted for individual cases, to avoid introducing individual bias into the comparisons.

The tract volumes were superimposed on the T1-weighted, volumetric sequences and then retrospectively reviewed in random order separately using Brainlab software by a neurosurgeon with 5 years of experience using tractography for surgical planning (blinded for all assessments). The neurosurgeon evaluated anatomic correctness and preference and whether the tract would alter surgical management. "Anatomically correct" implied that the tract matched known anatomy from previous surgical experience and reports of postmortem white matter dissection. ${ }^{22}$ "Different surgical management" implied that a tract changed the surgical approach or planned extent of resection. Finally, in blinded side-by-side comparison, the neurosurgeon determined whether he preferred 1 version of the tract.

The AF and CST length, FA, and volumes were extracted from the tract objects for standard and SMS diffusion tractography and then compared using paired-sample 2-tailed $t$ tests. Note that sample size was determined by practical considerations but was estimated a priori to require approximately 16 to detect a quantitative difference roughly equal to the anticipated variance of the data (ie, a Cohen D value of 1) with $95 \%$ statistical power. Confidence intervals also were calculated. The Dice similarity coefficient $(\mathrm{DSC})^{23}$ was used to measure the spatial overlap between standard (A) and SMS (B) tract volumes, where DSC(A, $\mathrm{B})=2(\mathrm{~A} \cap \mathrm{B}) /(\mathrm{A}+\mathrm{B})$ and $\cap$ is the intersection between the 2 volumes. Spatial misregistration from skull base geometric distortion was characterized by measuring the distance from the anterior margin of the conventional and SMS-derived CST tracts to the anterior margin of the cervicomedullary junction on coregistered volumetric $\mathrm{T} 1$ at

the foramen magnum.

\section{RESULTS}

\section{Clinical Diffusion Trace}

At 3T, 2-slice SMS acceleration with an altered diffusion-sampling scheme allowed us to reduce the TR by $59 \%$ (from 8.2 to 3.4 seconds), reduce diffusion volumes acquired $43 \%$ (from 14 to 8 ), and reduce overall scan time by $70 \%$ (from 141 to 45 seconds). There was an $18 \%$ decrease in perceived image quality of the SMS-accelerated scans $(P=.001)$. No difference in artifacts or diagnostic utility was detected with $95 \%$ confidence interval that true differences, if present, represented $<0.5$ decrement on the 5-point ordinal scale (Table 1). Representative clinical cases are provided in Figs 1 and 2 for readers to compare sequence results. Raters perceived subtle, lower SNR in the posterior fossa on SMS images (16\% absolute quantitative reduction, $P=.156$, with a $95 \%$ confidence interval to detect an SNR reduction of $\geq 38 \%$ ), but this remained acceptable for diagnostic use in ratings and subsequent clinical cases (Fig 2). Both readers noted a fat-saturation artifact in 1 subject undergoing SMS (Fig 1C); however, the diagnostic utility of this scan was not compromised. Raters did not observe increased T1-weighting to the SMS diffusion trace images; the TR of $3400 \mathrm{~ms}$ remained 


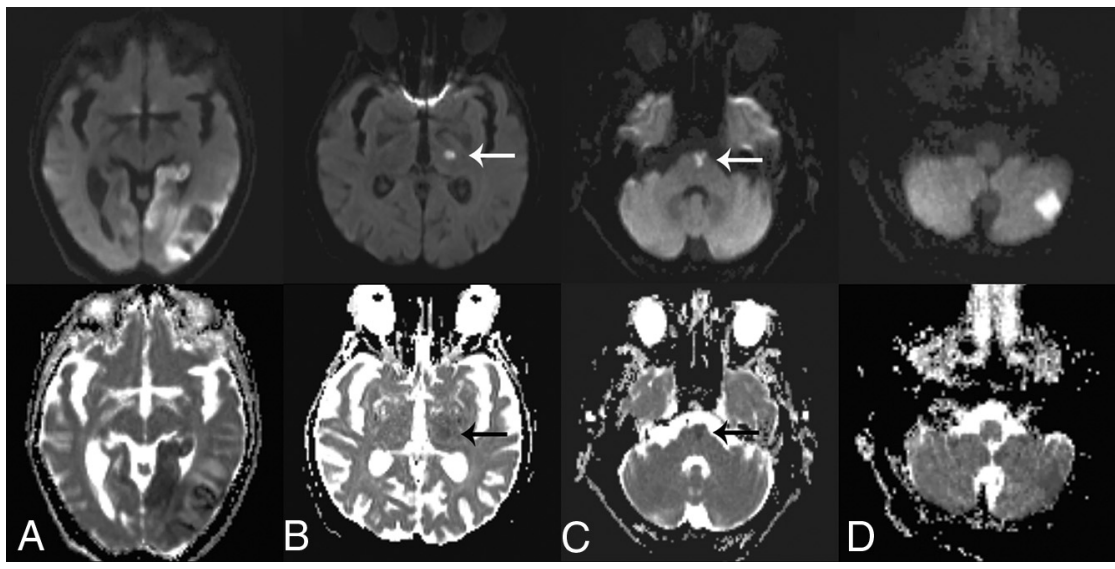

FIG 2. Examples of 2-slice SMS-accelerated diffusion trace and ADC maps of 4 different clinical patients. Large acute left-occipital infarct $(A)$ contrasted with a small subacute left-thalamic infarct $(B)$ (arrows). Raters suggested subtle SNR reductions for the posterior fossa using SMS (Table 1); however, small infarcts in the brainstem $(C)$ and cerebellum $(D)$ remain well-visualized.

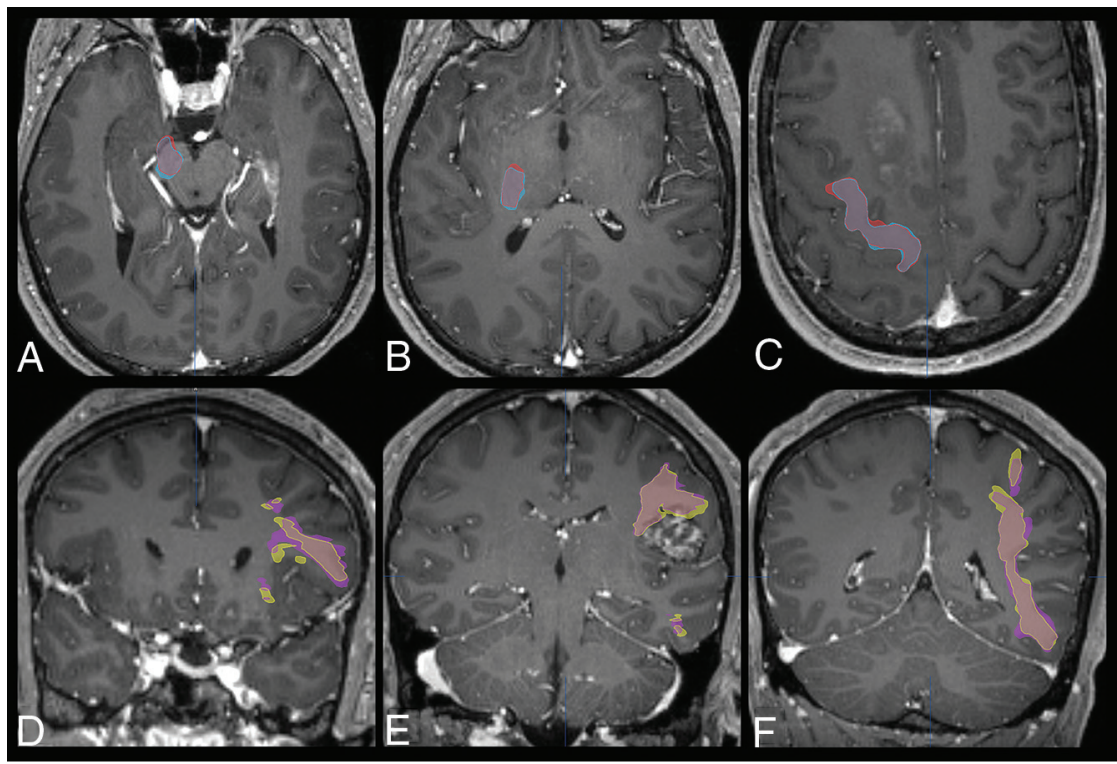

FIG 3. Overlap of diffusion tractography between standard and SMS-acquired data in 2 selected patients. Upper Row: A 56-year-old man with a multifocal right-cerebral hemisphere glioma. The right corticospinal tract volumes are shown in blue (conventional) and red (SMS) at the levels of the cerebral peduncle $(A)$, posterior limb of the internal capsule $(B)$, and precentral gyrus $(C)$. The DSC between standard and SMS tract volumes is 0.76. Lower Row: A 36-year-old man with a left opercular cavernous malformation. The left-arcuate fasciculus volumes are shown in purple (conventional) and yellow (SMS) at the levels of the anterior and posterior frontal projections ( $D$ and $E$ ) and genu $(F)$. There are slight differences in the edge of the visualized tracts abutting the superior border of the cavernous malformation $(E)$, but these differences would not affect selection of the surgical corridor, and in both situations, the neurosurgeon would be cautious in approaching the superior margin of the lesion. The DSC between standard and SMS tract volumes is 0.75 .

$3 \times$ the reported T1-relaxation times for gray and white matter at $3 \mathrm{~T} .^{24}$ There was no difference in the ADC values of the minor forceps or frontal horn CSF of standard compared with SMS diffusion images ( $95 \%$ confidence to exclude no greater than a $7 \%$ difference). There were no callbacks or nondiagnostic diffusion scans attributed to SMS during the subsequent 10 months after widespread adoption.
Rater agreement for artifacts, image quality, and diagnostic utility were $60 \%$, $62 \%$, and $76 \%$, respectively. The Cohen $\kappa$ values for interrater reliability were $0.216,0.363$, and 0.413 , respectively; these may underestimate agreement due to the first kappa paradox ${ }^{25}$ because ratings of 3 or 4 represented most scores. Reader agreement for artifacts, image quality, and diagnostic utility became $100 \%, 88 \%$, and $96 \%$ respectively if 3 and 4 ordinal scores from raters were collapsed to 1 value. While it was not part of the rater assessment, a similar 2-slice SMS-accelerated diffusion sequence was implemented on a newer 1.5T MRI scanner with a 20-channel coil (Magnetom Aera; Siemens) at the same hospital, reducing scan time from 165 to $65 \mathrm{~ms}$ (Online Supplemental Data); for reader interest, additional clinical examples of SMSaccelerated diffusion images at both field strengths are provided in the Online Supplemental Data. Note that full protocol parameters for $1.5 \mathrm{~T}$ and 3T MR imaging scanners will be provided to interested readers by contacting the communicating author.

\section{Diffusion Tractography}

2-slice SMS acceleration reduced the TR $34 \%$ to 4.7 seconds and reduced overall acquisition time $22 \%$ to 5.9 minutes without visual differences in tractography (Fig 3) or quantitative tractography differences for either the CST or AF ipsilateral to clinical lesions (Table 2). The mean DSCs between standard and SMS tract volumes were $0.78(\mathrm{SD}, 0.03)$ and 0.71 (SD, 0.05) for the CST and AF tracts, respectively ( $n=13$ subjects). The SMS-acquired CST demonstrated similar spatial misregistration at the skull base (Table 2). The Online Supplemental Data demonstrate reproducibility for CST and AF tracts in the same subject scanned twice 30 days apart.

The neurosurgeon rated 25 of 26 (96\%) SMS-generated tracts to be anatomically correct. The exception was the AF in case 11; however, both the conventional and SMS tracts were deemed equally nonanatomic in this case. This outcome was attributed to a porencephalic cavity, which made the standardized 2-ROI tractography approach ineffective and not from any inherent deficiency of the SMS acquisition. Tractography from SMS diffusion was considered equivalent or 
Table 2: Quantitative comparison of DTI tractography for the corticospinal tract and arcuate fasciculus ipsilateral to the lesion in 13 subjects $^{a}$

\begin{tabular}{|c|c|c|c|c|c|}
\hline Diffusion Technique & Standard & SMS & Mean Individual Difference ${ }^{b}$ & 95\% Cl for Difference & $P$ Value \\
\hline \multicolumn{6}{|l|}{ Corticospinal tract } \\
\hline Volume (mL) & $30.7[S D, 9.1]$ & $32.9[\mathrm{SD}, 9.7]$ & $-2.3[\mathrm{SD}, 4.0]$ & -9.4 to +5.0 & $.062^{c}$ \\
\hline Length (mm) & $125.2[S D, 13.1]$ & $124.0[\mathrm{SD}, 15.0]$ & $1.2[\mathrm{SD}, 7.0]$ & -9.6 to +12.0 & $.537^{\mathrm{c}}$ \\
\hline Mean FA & $0.45[S D, 0.04]$ & $0.46[S D, 0.04]$ & $-0.01[\mathrm{SD}, 0.02]$ & -0.04 to +0.02 & $.076^{\mathrm{c}}$ \\
\hline \multicolumn{6}{|l|}{ Arcuate fasciculus } \\
\hline Volume $(\mathrm{mL})$ & $25.3[S D, 5.3]$ & $26.0[S D, 6.7]$ & $-0.7[S D, 3.1]$ & -5.3 to +3.9 & $.438^{\mathrm{c}}$ \\
\hline Length (mm) & $77.4[\mathrm{SD}, 7.1]$ & $76.5[\mathrm{SD}, 6.7]$ & $0.9[S D, 4.6]$ & -4.4 to +6.2 & $.482^{\mathrm{c}}$ \\
\hline Mean FA & $0.36[S D, 0.04]$ & 0.36 [SD, 0.04] & $0.00[\mathrm{SD}, 0.01]$ & -0.03 to +0.03 & $1.000^{c}$ \\
\hline Spatial misregistration $(\mathrm{mm})^{\mathrm{d}}$ & $3.4[\mathrm{SD}, 1.6]$ & $2.8[\mathrm{SD}, 1.0]$ & $0.6[\mathrm{SD}, 1.3]$ & -0.4 to +1.6 & $.135^{c}$ \\
\hline
\end{tabular}

${ }^{a}$ Data are mean (SD) or $95 \% \mathrm{Cl}$.

${ }^{\mathrm{b}}$ Difference between measurements in the same individual (standard value minus SMS value).

'Paired-sample 2-tailed $t$ test.

${ }^{\mathrm{d}}$ Difference between the anterior margin of the cervicomedullary junction at the foramen magnum depicted by volumetric T MR imaging versus CST diffusion tractography.

better in 18 of 26 (69\%) tracts generated. Reasons for preferring one tract over its counterpart included the following: more robust tract, less spurious fibers, less discontinuities in volume, and less spurious extension into the extracranial soft tissues (removing the latter is normally routine, but not in this investigation to avoid introducing bias). All CST pairs did not change the surgical management (approach and aggressiveness). All AF pairs had no change in surgical approach. Eleven of 13 (85\%) AF pairs would have equivalent aggressiveness of resection. The standard AF tract in case 13 was considered a better anatomic representation of projections of the left-frontal operculum adjacent to a left-frontal oligodendroglioma that would result in a less aggressive resection. The SMS AF tract in case 5 was considered a more robust tract adjacent to the cavernous malformation, which would result in a less aggressive resection.

\section{DISCUSSION}

SMS-accelerated neuroradiology protocol diffusion acquisitions appear safe and clinically feasible without large differences in image quality when assessed by 2 neuroradiologists. Raters perceived mild decreases in the SNR of diffusion trace images, particularly in the posterior fossa structures, but characterization of pathologies of different sizes and in different anatomic locations appeared similar between standard and SMS-accelerated diffusion. Aggressive 2slice-accelerated SMS with 3T MR imaging and a standard 20-channel coil reduced our standard diffusion trace scan time approximately $70 \%$, saving 1.5 minutes per scan. Yokota et $\mathrm{al}^{26}$ reported a $41 \%$ scan time reduction using $3 \mathrm{~T}$ MR imaging and 2slice SMS acceleration with 2-mm isotropic resolution, but their final scan time of the SMS-accelerated sequence was still $3 \times$ longer than our current data. ${ }^{26}$ Given that diffusion imaging is ubiquitous to all neuroradiology protocols, the 1.5 -minute time savings obtained here would return sufficient time theoretically for an additional 3 scans per day per magnet at our primary outpatient facility (estimation based on $\sim 67.5$ minutes saved; the facility is open 15 hours per day, with 20 -minute slots and $100 \%$ neuroradiology head protocols). A motion-free 45-second diffusion scan should facilitate more rapid decision-making in acute stroke, decrease motion degradation in patients who are less MR imaging-tolerant, and create more time for additional MR imaging sequences. An additional motivation for our center was to create more time within the scheduled slots to clean the magnet between patients during the early stages of the coronavirus 19 pandemic.

SMS-accelerated diffusion tractography was considered anatomically equivalent by an experienced neurosurgeon without altering intended surgical management or the aggressiveness of resection. The tractogram volume agreement was good: mean DSCs of 0.78 and 0.71 for CST and AF, respectively. An imperfect DSC may occur due to low spatial resolution ( $\sim 3-\mathrm{mm}$ isotropic voxels), SNR differences, patient movement (or other causes of misregistration), and/or different spurious tracts not actively edited from the data. In our clinical practice, neuroradiologists freely and subjectively manipulate the tractography parameters more on an individual basis than permitted in this study design to accomplish the goals of presurgical planning. Yokota et $\mathrm{al}^{26}$ reported that CST tractography appeared similar to radiologists with or without 2slice SMS acceleration, but most of the data were from nonsurgical patients and not blindly evaluated by a neurosurgeon experienced using tractography. Here, SMS acquisitions in preoperative cases could reduce tractography scan times by $\sim 2$ minutes; these studies tend to be long due to the need for coregistered fMRI, tractography, and 3D structural MR imaging. Even more time savings might be possible with 3- or 4-fold slice acceleration using array coils with more receive elements (eg, a 64-channel coil), less angular or $q$-space resolution than in our current institutional protocol, or improved monopolar imaging gradients that reduce TE (and subsequently scan time).

Besides increased throughput on busy clinical MR imaging scanners, SMS acceleration can also be used to increase spatial resolution of clinical diffusion MR imaging protocols; this potential was not emphasized in the current study but could be the subject of future work. SMS-accelerated diffusion images for the current study still had TRs (3200 or $4700 \mathrm{~ms}$ ) more than $3 \times$ the T1-relaxation times of gray and white matter at $3 \mathrm{~T}^{24}$ More aggressive reductions of TR are possible, particularly with 3- or 4-slice acceleration, which may introduce T1-weighting to diffusion trace images. In preliminary experiments, shortening the TR below $2500 \mathrm{~ms}$ also introduced more substantial signal loss and increased the frequency of peripheral nerve stimulation reported by volunteer subjects. With SMS, unsaturated fat signal associated with echo-planar acquisitions can alias into all simultaneously acquired slices and result in spurious tractography. While SMS diffusion is now FDA-approved, readers 
should carefully adapt SMS acquisitions with or without acceleration to their local diffusion protocols.

This study has limitations. Due to practical limitations on the time required for detailed assessment by radiologists and a neurosurgeon, the study is underpowered to exclude smaller quantitative differences between sequences (see confidence intervals in Tables 1 and 2). The SMS-accelerated diffusion sequences used here are derived from a single large academic center (New York University) but may differ from other radiology practice locations and settings. Scan time reductions were not just from 2-slice SMS acceleration but also the elimination of the $b=500 \mathrm{~s} / \mathrm{mm}^{2}$ images. The external validity of our results is supported by another report of SMS-accelerated diffusion equivalence, albeit with less time savings, using a different sequence (2- $\mathrm{mm}$ isotropic resolution, $b=0$ and $1000 \mathrm{~s} / \mathrm{mm}^{2}$ with 10 directions, a final time of 128 seconds, which was $41 \%$ faster than the previous protocol). ${ }^{26}$ Hence changes in time savings, perceived image quality, artifacts, and diagnostic utility will vary depending on the local accepted protocol and how it is modified. Another limitation was that tractography results were reviewed retrospectively so that the position of the SMS-derived tracts could not be correlated with operative findings (the standard diffusion data were instead used as an index standard). Intraoperative verification of tractography with or without SMS acceleration in patients requiring surgery is not trivial to obtain, but it could be the subject of a future study. There are additional methods for accelerating diffusion acquisitions that are being translated into clinical practice, including compressed sensing ${ }^{27}$ and image denoising with ${ }^{28}$ or without deep learning. ${ }^{29}$ These techniques are not readily available for clinical implementation, but direct comparison of these diffusion-acceleration techniques and/or their combination also could be the subject of future study.

The characterization of nervous tissue microstructure with advanced diffusion models in clinical patients has been hampered by the time-intensive high-angular and $q$-space acquisition requirements that SMS acceleration helps overcome. ${ }^{30}$ For instance, as part of an ongoing National Institutes of Healthfunded study, we have used SMS to acquire high-spatial and -angular resolution, multi-b-shell diffusion data from 137 patients with multiple sclerosis. ${ }^{31}$ We also have used SMS-accelerated acquisitions to generate probabilistic tractographyderived contrast of internal brainstem anatomy. ${ }^{32}$ SMS diffusion can complement other means of accelerating clinical neuroradiology MR imaging protocols like compressed sensing, ${ }^{33}$ synthetic MR imaging, ${ }^{34}$ or MR fingerprinting ${ }^{35}$ that have, thus far, been more limited at producing clinically acceptable accelerations or substitutions for conventional diffusion-weighted sequences. $^{36}$

\section{CONCLUSIONS}

Using standard 3T MR imaging hardware and coils, 2-slice SMS acceleration can reduce routine diffusion MR imaging and diffusion tractography acquisition times in clinical patients without compromising image quality, clinical interpretation, or surgical planning. SMS acceleration also should increase the feasibility of translating high spatial and angular resolution diffusion acquisitions to clinical patients.

\section{ACKNOWLEDGMENTS}

The authors thank Matt Young and Benjamin Cohen for performing initial clinical evaluations of the SMS diffusion sequence.

Disclosures: Donato Pacione - UNRELATED: Consultancy: 2nd.MD consulting. Yvonne W. Lui-UNRELATED: Grants/Grants Pending: National Institutes of Health, Lowenstein Foundation, Department of Defense, National Science Foundation.* Els Fieremans_UNRELATED: Board Membership: MICroStructure Imaging, Comments: no money paid; Grants/Grants Pending: National Institutes of Health, Comments: R01 NS039135, P41 EB017183*; Royalties: GE Healthcare, Comments: patent licensing; Stock/ Stock Options: MICroStructure Imaging, Comments: no money paid. Timothy M. Shepherd-RELATED: Grant: National Institutes of Health, Comments: 1) National Institute of Aging (National Institutes of Health 1K23 AG048622-01), and 2) National Institutes of Health-National Institute of Biomedical Imaging and Bioengineering Biomedical technology resource center (Grant P41EB017183)*; UNRELATED: Board Membership: MICroStructure Imaging, Comments: founder and equity holder. *Money paid to the institution.

\section{REFERENCES}

1. Schaefer PW, Grant PE, Gonzalez RG. Diffusion-weighted MR imaging of the brain. Radiology 2000;217:331-45 CrossRef Medline

2. Barajas RF Jr, Rubenstein JL, Chang JS, et al. Diffusion-weighted MR imaging derived apparent diffusion coefficient is predictive of clinical outcome in primary central nervous system lymphoma. AJNR Am J Neuroradiol 2010;31:60-66 CrossRef Medline

3. Nemeth AJ, Henson JW, Mullins ME, et al. Improved detection of skull metastasis with diffusion-weighted MR imaging. AJNR Am J Neuroradiol 2007;28:1088-92 CrossRef Medline

4. Murata T, Shiga Y, Higano S, et al. Conspicuity and evolution of lesions in Creutzfeldt-Jakob disease at diffusion-weighted imaging. AJNR Am J Neuroradiol 2002;23:1164-72 Medline

5. Rilling JK, Glasser MF, Preuss TM, et al. The evolution of the arcuate fasciculus revealed with comparative DTI. Nat Neurosci 2008;11:42628 CrossRef Medline

6. Wu JS, Zhou LF, Tang WJ, et al. Clinical evaluation and follow-up outcome of diffusion tensor imaging-based functional neuronavigation: a prospective, controlled study in patients with gliomas involving pyramidal tracts. Neurosurgery 2007;61:935-48; discussion 948-49 CrossRef Medline

7. Berman JI, Berger MS, Chung SW, et al. Accuracy of diffusion tensor magnetic resonance imaging tractography assessed using intraoperative subcortical stimulation mapping and magnetic source imaging. J Neurosurg 2007;107:488-94 CrossRef Medline

8. Jellison BJ, Field AS, Medow J, et al. Diffusion tensor imaging of cerebral white matter: a pictorial review of physics, fiber tract anatomy, and tumor imaging patterns. AJNR Am J Neuroradiol 2004;25:356-69 Medline

9. Novikov DS, Fieremans E, Jespersen SN, et al. Quantifying brain microstructure with diffusion MRI: theory and parameter estimation. NMR Biomed 2019;32:e3998 CrossRef Medline

10. Tuch DS. Q-ball imaging. Magn Reson Med 2004;52:1358-72 CrossRef Medline

11. Wedeen VJ, Hagmann P, Tseng WY, et al. Mapping complex tissue architecture with diffusion spectrum magnetic resonance imaging. Magn Reson Med 2005;54:1377-86 CrossRef Medline

12. Jensen J, Helpern J, Ramani A, et al. Diffusional kurtosis imaging: the quantification of non-Gaussian water diffusion by means of magnetic resonance imaging. Magn Reson Med 2005;53:1432-40 CrossRef Medline

13. Larkman DJ, Hajnal JV, Herlihy AH, et al. Use of multicoil arrays for separation of signal from multiple slices simultaneously excited. $J$ Magn Reson Imaging 2001;13:313-17 CrossRef Medline

14. Setsompop K, Cohen-Adad J, Gagoski BA, et al. Improving diffusion MRI using simultaneous multi-slice echo planar imaging. Neuroimage 2012;63:569-80 CrossRef Medline

15. Setsompop K, Gagoski BA, Polimeni JR, et al. Blipped-controlled aliasing in parallel imaging for simultaneous multislice echo 
planar imaging with reduced g-factor penalty. Magn Reson Med 2012;67:1210-24 CrossRef Medline

16. Cauley SF, Polimeni JR, Bhat $\mathrm{H}$, et al. Interslice leakage artifact reduction technique for simultaneous multislice acquisitions. Magn Reson Med 2014;72:93-102 CrossRef Medline

17. Obele CC, Glielmi C, Ream J, et al. Simultaneous multislice accelerated free-breathing diffusion-weighted imaging of the liver at 3T. Abdom Imaging 2015;40:2323-30 CrossRef Medline

18. Lau AZ, Tunnicliffe EM, Frost R, et al. Accerlerated human cardiac diffusion tensor imaging using simultaneous multislice imaging. Magn Reson Med 2015;73:995-1004 CrossRef Medline

19. Filli L, Piccirelli M, Kenkel D, et al. Simultaneous multislice echo planar imaging with blipped controlled aliasing in parallel imaging results in higher acceleration: a promising technique for accelerated diffusion tensor imaging of skeletal muscle. Invest Radiol 2015;50:456-63 CrossRef Medline

20. Gudbjartsson H, Patz S. The Rician distribution of noisy MRI data. Magn Reson Med 1995;34:910-14 CrossRef Medline

21. Catani $M$, Howard RJ, Pajevic $S$, et al. Virtual in vivo interactive dissection of white matter fasciculi in the human brain. Neuroimage 2002;17:77-94 CrossRef Medline

22. Ludwig E, Klingler J. Atlas Cerebri Humani. Karger; 1956

23. Dice LR. Measures of the amount of ecologic association between species. Ecology 1945;26:297-302 CrossRef

24. Wansapura JP, Holland SK, Dunn RS, et al. NMR relaxation times in the human brain at 3.0 Tesla. J Magn Reson Imaging 1999;9:53138 CrossRef Medline

25. Feinstein AR, Cicchetti DV. High agreement but low kappa, I: the problems of two paradoxes. J Clin Epidemiol 1990;43:543-49 CrossRef Medline

26. Yokota H, Sakai K, Tazoe J, et al. Clinical feasibility of simultaneous multi-slice imaging with blipped-CAIPI for diffusion-weighted imaging and diffusion-tensor imaging of the brain. Acta Radiol 2017;58:1500-10 CrossRef Medline
27. Ning L, Setsompop K, Michailovich O, et al. A compressed-sensing approach for super-resolution reconstruction of diffusion MRI. Inf Process Med Imaging 2015;24:57-68 CrossRef Medline

28. Kawamura M, Tamada D, Funayama S, et al. Accelerated acquisition of high-resolution diffusion-weighted imaging of the brain with a multi-shot echo-planar sequence: deep-learning-based denoising. Magn Reson Med Sci 2021;20:99-105 CrossRef Medline

29. Veraart J, Novikov DS, Christiaens D, et al. Denoising of diffusion MRI using random matrix theory. Neuroimage 2016;142:394-406 CrossRef Medline

30. Novikov DS, Kiselev VG, Jespersen SN. On modeling. Magn Reson Med 2018;79:3172-93 CrossRef Medline

31. Gillingham N, Ades-Aron B, Conti B, et al. Normal appearing white matter damage assessed with diffusion MRI, but not T2 lesion load, correlates with disability and disease duration in multiple sclerosis. In: Proceedings of the International Society for Magnetic Resonance in Medicine Workshop on Quantitative MRI in White Matter Disorders: Useful, Usable, Used? Vancouver, British Columbia, Canada. February 7-10, 2017:15

32. Hoch MJ, Chung S, Ben-Eliezer N, et al. New clinically-feasible 3T MRI protocol to discriminate internal brain stem anatomy. AJNR Am J Neuroradiol 2016;37:1058-65 CrossRef Medline

33. Lustig M, Donoho D, Pauly JM. Sparse MRI: the application of compressed sensing for rapid MR imaging. Magn Reson Med 2007;58:1182-95 CrossRef Medline

34. Warntjes JB, Dahlqvist O, Lundberg P. Novel method for rapid, simultaneous $\mathrm{T} 1, \mathrm{~T}^{\star}$, and proton density quantification. Magn Reson Med 2007;57:528-37 CrossRef Medline

35. Ma D, Gulani V, Seiberlich N, et al. Magnetic resonance fingerprinting. Nature 2013;495:187-92 CrossRef Medline

36. Powell HW, Parker GJ, Alexander DC, et al. Hemispheric asymmetries in language-related pathways: a combined functional MRI and tractography study. Neuroimage 2006;32:388-99 CrossRef Medline 